\title{
Chest reconstruction using a custom-designed polyethylene 3D implant after resection of the sternal manubrium
}

This article was published in the following Dove Press journal:

OncoTargets and Therapy

17 August 2017

Number of times this article has been viewed

\author{
Joanna Lipińska' \\ Leszek Kutwin' \\ Marcin Wawrzycki' \\ Leszek Olbrzymek ${ }^{2}$ \\ Sławomir Jabłoński' \\ 'Department of Thoracic Surgery, \\ General and Oncological Surgery, \\ Medical University of Lodz. \\ ${ }^{2}$ Ledo, Lodz, Poland
}

Correspondence: Joanna Lipińska Department of Thoracic Surgery, General and Oncological Surgery, Medical University of Lodz, 64 m.65 Dąbrowskiego Street, 93-208 Lodz, Poland

Tel +4842639352I

Fax +48 426333890

Email joasialipinska@yahoo.com
Introduction: Resection of manubrium or body of the sternum is associated with a necessity of chest wall reconstruction. Large sternal defects require the use of different types of implants to ensure acceptable esthetic effect for the patient and chest stabilization.

Aim: The purpose of this case report is to present a novel method of reconstruction of manubrium removed due to renal cancer metastasis to the sternum.

Case: We present the case of a patient, who had underwent right nephrectomy for clear cell kidney cancer, diagnosed with a metastatic tumor in the sternum resulting in destruction of manubrium. The patient undergone tumor resection with primary reconstruction with an individual prosthesis. Sternal defect was filled with a personalized, computed tomography scan-based 3D-milled implant made of polyethylene.

Results: Sternal reconstruction was uneventful. The patient endured surgery well, and has been under surveillance in outpatient clinic, without any respiration disorders, implant movement or local recurrence.

Conclusion: Custom-designed sternal implants created by 3D technique constitute an interesting alternative for previous methods of filling defects after resection of a tumor in this location.

Keywords: 3D-milled implant, thoracoplasty, reconstructive surgery, chest reconstruction, sternal metastasis, sternal implant, sternal tumor

\section{Introduction}

Primary malignant and benign neoplasms, metastatic neoplasms and rarely congenital and acquired deformations can be indications for sternum resection. The skeletal system constitutes the second most common location of malignant neoplasm metastases from other organs. Predilection for metastases formation in bones is characteristic of pulmonary, prostatic and chest carcinoma, with a prevalent location in axial skeleton bones. It is assumed that metastatic changes in bones are found almost 100 times more often than primary ones. The sternum is a rare location of metastases, ${ }^{1}$ and chest carcinoma is considered as their main source. ${ }^{2}$ Symptoms of metastasis of tumors to the sternum are perceptible pathological mass in sternum projection, tenderness in the area of change and its deformation, pains while moving or resting and pathological fractures. ${ }^{1}$ As a rule, oncological history confirms a primary tumor or a surgical resection undergone earlier or a combined treatment. In such situation, a diagnostic examination can indicate the coexistence of additional metastatic changes in other organs. If a diagnosed metastasis is the sole metastatic change in the body and there is a possibility of primary focus control, its resection, giving the chance for long-standing survival, should be the treatment of choice. 


\section{Case description}

In November 2015, a 52-year-old male patient with a sternal manubrium tumor, burdened by an extended oncological history, was admitted to the Thoracic Surgery Clinic, General and Oncological Clinic, Medical University of Lodz. The patient came to the Thoracosurgical Outpatient Clinic for the first time in July 2015 complaining of sternal pains. The chest computed tomography (CT) scan revealed a right lung tumor with an extensive osteolytic change involving a soft tissue component in sternal manubrium, slightly widening beyond the back contour of the sternum. In the very same $\mathrm{CT}$ examination, an additional right kidney tumor was found. Positron emission tomography was performed (Figure 1) which revealed metabolically active changes (sternal tumor maximum standardized uptake value of fluorine 18 fluorodeoxyglucose $=3.5$, pulmonary tumor maximum standardized uptake value of fluorine 18 fluorodeoxyglucose $=13.6$ ). The patient denied other chronic diseases. His family history was positive as to neoplastic diseases - patient's father suffered from gastric carcinoma, and his grandfather from chronic myeloid leukemia. The patient underwent right nephrectomy in August 2015, and on the basis of histopathological examination, he was diagnosed with clear cell kidney cancer. Due to this, changes in the lungs and sternum were considered as metastatic. In September 2015, the patient was subjected to right upper lobectomy by classical anterior-lateral approach. Final histopathological examination, though, revealed squamous epithelial carcinoma of a lung. The situation implied that the change in the sternum can be a metastasis of one of the above-mentioned neoplasms. Due to massive bone destruction and the fact of not knowing the etiology of the sternal tumor, the multidisciplinary oncological and surgical team recommended the resection of manubrium.

On 10 November 2015, resection of sternal manubrium was performed along with reconstruction of an individually

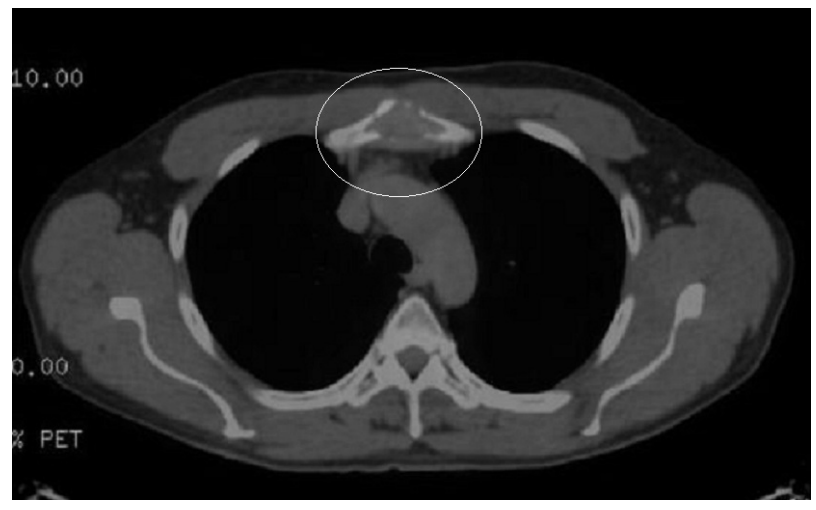

Figure I Positron emission tomography-computed tomography examination of metabolically active sternal tumor (white circle: maximum standardized uptake value of fluorine 18 fluorodeoxyglucose $=3.5$ ). dedicated implant made of high-molecular polyethylene. In brief, the surgical technique was as follows: The procedure was started with a longitudinal incision of the skin in the central line above the tumor. After dissection of the pectoral major muscle attachments, a tumor, of size $30 \times 30 \mathrm{~mm}$, was seen, exceeding the anterior lamina of the sternum. Costal cartilages I and II were dissected, and clavicles from sternalclavicular joints were relieved. Below the tumor, the sternum was dissected transversally maintaining the $3-\mathrm{cm}$ margin of a healthy bone. The lateral side of the tumor was surrounded by unchanged osseous tissue. Moving from the bottom to the top, the manubrium together with the pathological mass was removed. The material was sent immediately to histopathological examination, which gave the diagnosis of metastasis of a clear cell carcinoma. In the place of an extensive bone defect of the upper sternal part, an earlier prepared dedicated implant of sternal manubrium made of high-molecular polyethylene was placed. The shape and size of the implant were worked out based on a special software program including the data of the patient's CT scan. Dicom files from CT data were used for recreating the proper sternal shape in file ".stl", in "Invasalius" program.

Using SolidWorks program, solid form with "parasolisd. $\mathrm{xt}$ " extension was achieved in "NX CAM" program which allowed for generating special codes for numerically controlled milling machine. Processing tomography with consideration to a planned resection range and after designer's consultation with a surgeon, the final 3D implant was created (Figure 2). Next, using numerically controlled milling machine, the implant was made of certified polyethylene of ultrahigh molecular mass which is rigid and nonabsorbable.

The patient was informed about the purpose of the study, and written informed consent was obtained to have the case

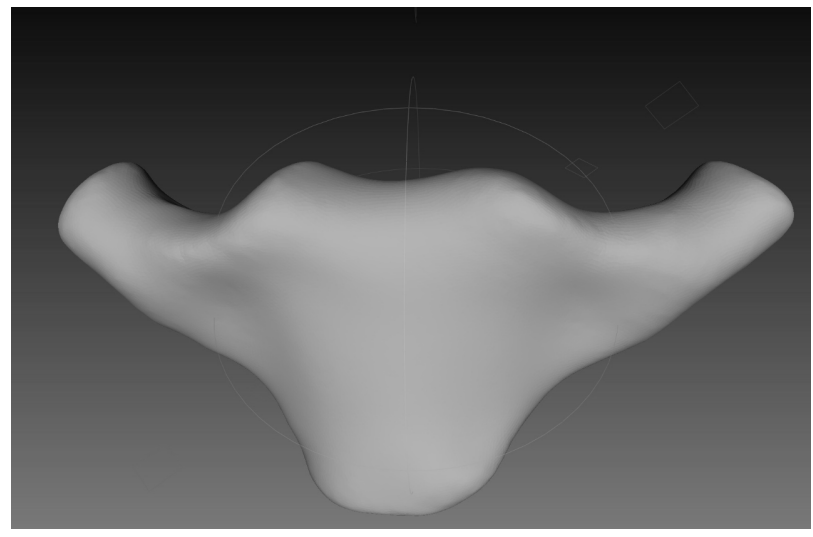

Figure 2 3D model of the implant prepared based on software program including the date of the patient's computed tomography scan. 
details and any accompanying images published. The study protocol was approved by the Bioethical Committee of the Medical University of Lodz.

Due to physical properties, the implant constituted a perfect base for anastomosis with bone scaffolding adjacent to sternal defect. The implant adjusted to the defect was fixed to the clavicles, sternum and ribs with the help of titanium rib plates (Figure 3). The material was prepared 7 days before the operation and subjected to sterilization. The size of the implant was slightly bigger than the removed sternal manubrium and had to be trimmed during the procedure using a sharp knife and surgical file. Full stabilization of shoulder girdle and anterior chest wall was achieved. After hemostasis control, pectoral muscles were stitched with each other. The patient endured the surgery well. On the second day after the surgery, control chest X-ray was performed; no pneumothorax or fluid in the pleural cavity was found. As observed on the photograph, the plates fixing the implant to ribs did not shift. On the next day, Redon drain was removed, and on the fourth day after the operation, the patient was discharged home in a good general condition. In a final histopathological description of resected change, the diagnosis of renal cancer metastasis to the sternum was confirmed. Clavicular margins, lower sternal and costal, were free of tumor cells. The skin sutures were removed after 10 days in dispensary. During next months of follow-up, the patient denied any pains related to the sternum, and returned to his profession as a teacher (Figure 4). No shoulder girdle deformity or disorder was noticed. Full range of motion in shoulder joint was conserved. Yet, in a further follow-up after 4 months, the patient was diagnosed with a next metastatic change, this time located in the left neck of the femur. The patient underwent alloplasty with inserting left hip prosthesis. Currently, 2 months after the surgery, the patient's condition is good, and he has been

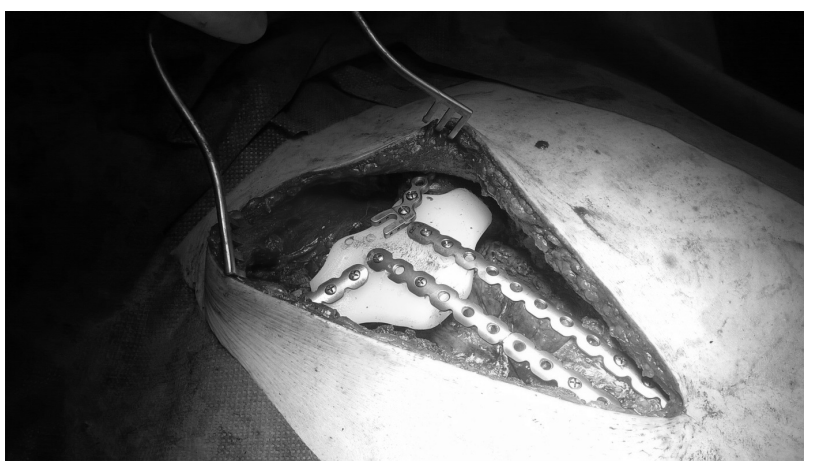

Figure 3 Intraoperative picture: 3D sternal implant filling the defect after tumor resection fixed to bone scaffolds by titanium plates.

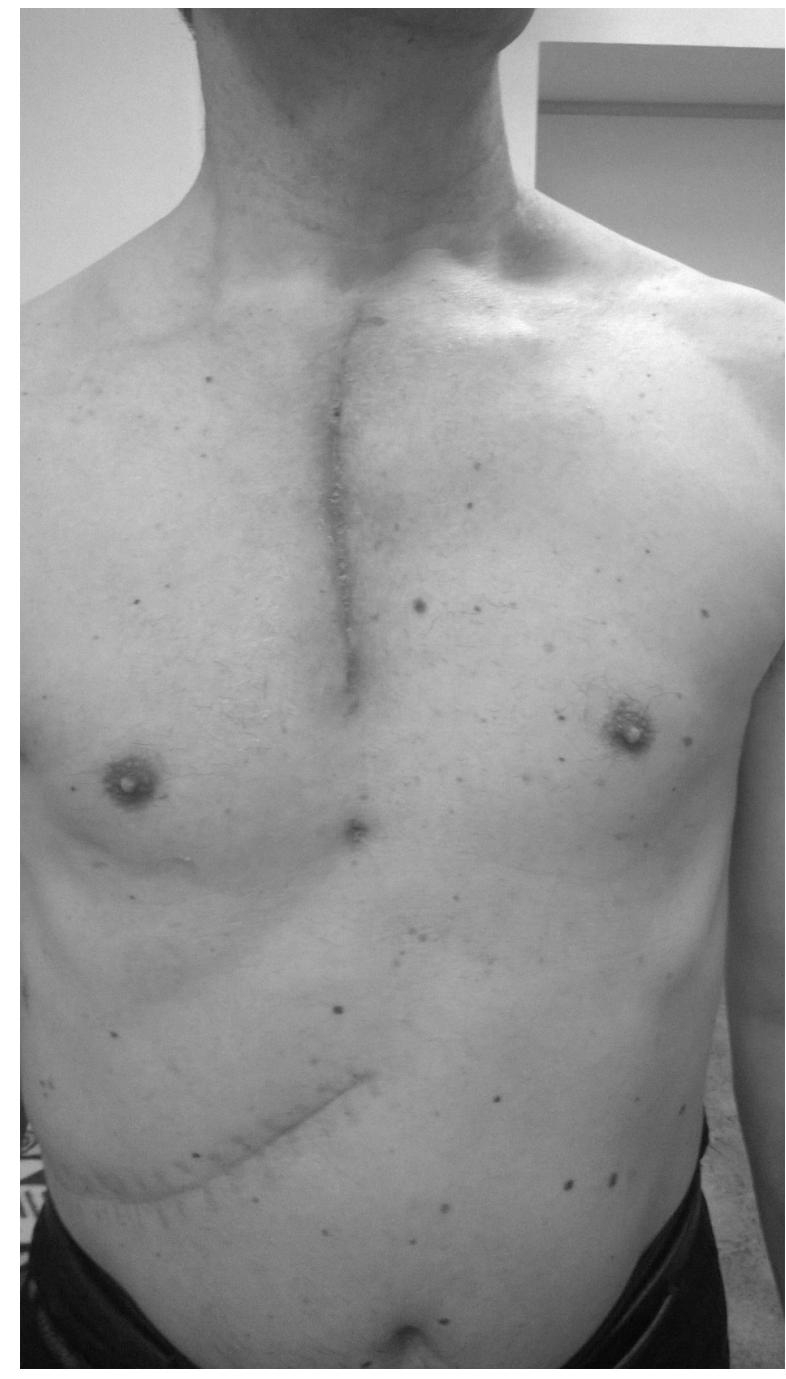

Figure 4 Skin scar 3 months after the operation.

under surveillance of the Thoracosurgical and Orthopedic Outpatient Clinic.

\section{Discussion}

Resection of the body or manubrium of the sternum requires chest wall reconstruction using a material which enables stabilization of the chest, ensures proper respiratory functions and protects organs in its interior. ${ }^{3}$ So far, this treatment scheme has not been established. There are no doubts as to the fact that every malignant tumor, apart from chemosensitive primary microcellular sarcoma (Ewing sarcoma, primary neuroectodermal sarcoma), should be removed entirely, ideally with the margin of $2-5 \mathrm{~cm}$ of healthy tissues. ${ }^{4}$ The choice of chest reconstruction depends, however, on the size of the defect and operator preferences. Defects of $<5 \mathrm{~cm}$ diameter do not usually require rigid implant reconstruction. ${ }^{5}$ In case of resection of big sternal fragments, it is 
recommended to substitute them with auto- or allogeneic material. The range of applied implants here is very wide. The most often used are methyl methacrylate implants ${ }^{3}$ or titanium plates ${ }^{6}$ coated with sealed prolene mesh from inside and outside of the chest wall (sandwich technique), Marlex, polytetrafluoroethylene or Vicryl. ${ }^{7}$ In addition, the following are also used: Ley's prostheses in the form of a titanium plate in the shape of a footprint fixed to the ribs with metal threads, ${ }^{8}$ sternum and rib fragments made of titanium in $3 \mathrm{D}$ technique, ${ }^{9,10}$ silicone implants made on the basis of a patient's chest print before the surgery (in this case, most complications are observed, like recurrent serous exudate up to $31 \%),{ }^{11}$ autografts of osseous tissue and muscle fragments ${ }^{6}$ and allografts of the sternal fragment from deceased donors. ${ }^{4}$ Implant should be resistant to forces acting on chest skeleton, plastic so as to adjust it according to the size of the sternum defect, translucent for X-rays ${ }^{7}$ and also made of a material which is not a breeding ground for bacterial flora. ${ }^{3}$ Polyethylene seems to be a good material for sternal implants because it is rigid and nonabsorbable, and does not react with bones, soft tissues or body liquids. It does not support the growth of any organism. It has proper rigidity, resistance and is impermeable for X-rays. It allows fixing to the metal plates (titanium, surgical steel) and bones using screws. It is however not recommended for joints as an endoprosthesis because it is prone to surface abrasion which can be harmful.

Reconstruction procedures after sternum resection are difficult and burdened with significant percentage of complications - estimated to be about 33\%-46\%. The most often observed are the complications associated with respiratory disorders. ${ }^{11}$ Other known complications connected with sternum implants are chronic pains, chest deformations, local pleural thickening, necrosis of used pedicle muscle lobes, ${ }^{6}$ mediastinitis and loosening of the implant mounting resulting in instability of the chest. Sternal implant fracture has also been reported. ${ }^{12}$ Predictive factors of increased risk of complications after chest reconstruction are patient's age $>60$ years old, size of the chest defect and simultaneous lung resection procedure. ${ }^{5}$

\section{Conclusion}

On the basis of the case presented here, it seems that custommade, printed or milled 3D sternal implants, made from biologically neutral synthetic materials produced in individual size using special computer programs with data from CT imaging, constitute interesting alternative for past methods of defect management after tumor resection in this location.
High-molecular polyethylene prosthesis fulfilled all requirements concerning a good filling for a missing fragment of a bone. After operation, no complications were observed either. To the authors' knowledge, the presented procedure was the first successful attempt of this kind of management of sternal defect with a 3D-milled implant in Poland and the second in Europe. Successive analogical procedures will allow for collecting materials more precisely defining disadvantages and advantages of such method of management of similar changes.

\section{Acknowledgment}

This work was supported administratively by the Medical University of Lodz, Poland.

\section{Disclosure}

The authors report no conflicts of interest in this work.

\section{References}

1. Ball E, Morris-Stiff G, Coxon M, Lewis MH. Mucinous adenocarcinoma presenting as an isolated sternal metastasis. World J Surg Oncol. 2007;5:105.

2. Kluiber R, Bines S, Bradley C, Faber LP, Witt TR. Major chest wall resection for recurrent breast carcinoma. Am Surg. 1991;57(8):523-529; discussion 529-530.

3. Lee SY, Lee SJ, Lee CS. Sternum resection and reconstruction for metastatic renal cell cancer. Int J Surg Case Rep. 2011;2(4):45-46.

4. Zhao Y, Peng C, Liu Y, Hao Y, Zhao X, Chong B. Clinical study of allogeneic graft reconstruction for sternum tumor. Exp Clin Transplant. 2016;14(3):353-357.

5. Weyant MJ, Bains MS, Venkatraman E, et al. Results of chest wall resection and reconstruction with and without rigid prosthesis. Ann Thorac Surg. 2006;81(1):279-285.

6. Haraguchi S, Hioki M, Hisayoshi T, et al. Resection of sternal tumors and reconstruction of the thorax: a review of 15 patients. Surg Today. 2006;36(3):225-229.

7. Mansour KA, Thourani VH, Losken A, et al. Chest wall resections and reconstruction: a 25-year experience. Ann Thorac Surg. 2002;73(6): 1720-1725; discussion 1725-1726.

8. Pedersen TA, Pilegaard HK. Reconstruction of the thorax with Ley prosthesis after resection of the sternum. Ann Thorac Surg. 2009;87(4): e31-e33.

9. Aranda JL, Jiménez MF, Rodríguez M, Varela G. Tridimensional titanium-printed custom-made prosthesis for sternocostal reconstruction. Eur J Cardiothoracic Surg. 2015;48(4):e92-e94.

10. Anderson CJ, Spruiell MD, Wylie EF, et al. A technique for pediatric chest wall reconstruction using custom-designed titanium implants: description of technique and report of two cases. J Child Orthop. 2016; 10(1):49-55.

11. Snel BJ, Spronk CA, Werker PM, van der Lei B. Pectus excavatum reconstruction with silicone implants: long-term results and a review of the English-language literature. Ann Plast Surg. 2009;62(2):205-209.

12. Yordanov D, Assenov R, Badarov T, Kesov G, Yordanov V. A unique case of fractured titanium implant after sternal resection. J Cardiothorac Surg. 2013;8(Suppl 1):O77. 
OncoTargets and Therapy

\section{Publish your work in this journal}

OncoTargets and Therapy is an international, peer-reviewed, open access journal focusing on the pathological basis of all cancers, potential targets for therapy and treatment protocols employed to improve the management of cancer patients. The journal also focuses on the impact of management programs and new therapeutic agents and protocols on

patient perspectives such as quality of life, adherence and satisfaction The manuscript management system is completely online and includes a very quick and fair peer-review system, which is all easy to use. Visit http://www.dovepress.com/testimonials.php to read real quotes from published authors.

Submit your manuscript here: http://www.dovepress.com/oncotargets-and-therapy-journal 